International Journal of Modern Physics E

(C) World Scientific Publishing Company

\title{
Signatures for Black Hole production from hadronic observables at the Large Hadron Collider
}

\author{
Thomas J. Humanic \\ Department of Physics, The Ohio State University \\ Columbus, Ohio, 43210 \\ humanic@mps.ohio-state.edu \\ Benjamin Koch and Horst Stöcker \\ Institut für Theoretische Physik, J. W. Goethe-Universität \\ Max von Laue Strasse 1, 60438 Frankfurt am Main, Germany \\ koch@th.physik.uni-frankfurt.de/stoecker@th.physik.uni-frankfurt.de
}

\begin{abstract}
The concept of Large Extra Dimensions (LED) provides a way of solving the Hierarchy Problem which concerns the weakness of gravity compared with the strong and electroweak forces. A consequence of LED is that miniature Black Holes (mini-BHs) may be produced at the Large Hadron Collider in $p+p$ collisions. The present work uses the CHARYBDIS mini-BH generator code to simulate the hadronic signal which might be expected in a mid-rapidity particle tracking detector from the decay of these exotic objects if indeed they are produced. An estimate is also given for $\mathrm{Pb}+\mathrm{Pb}$ collisions.
\end{abstract}

\section{Introduction}

The Hierarchy Problem in particle physics is concerned with the question of why gravity is so weak compared with the other forces in nature, or posed another way, why the scale for gravity, i.e. the Planck mass at $\sim 10^{19} \mathrm{GeV}$, is so much larger than the scales for the other forces in nature, the strong force with a Lund string model scale of $\sim 1 \mathrm{GeV} / \mathrm{fm}$ and the electro-weak force characterized by the mass of the $\mathrm{W}$ and $\mathrm{Z}$ bosons, $\sim 100 \mathrm{GeV}$. Several solutions have been proposed to solve this problem, such as 1) Supersymmetry in which bosons and fermions are symmetric and which unifies the strong and electro-weak forces at a scale just below the Planck scale, 2) String Theory in which elementary particles are represented by higher dimensional strings, e.g. 11 dimensional, and which is a theory of quantum gravity thought to be valid up to the Planck scale and beyond, and 3) the concept of Large Extra Dimensions (LED) which also assumes space-time has a higher dimensionality than the normal $3+1$ dimensions $^{1,2}$. The present study will be carried out in the framework of the LED model of Arkani-Hamed, Dimopoulos, and Dvali ${ }^{1,3}$, called the ADD model, so some further discussion of the model is given. This model has produced a great deal of interest in the literature, and there exist many papers that discuss it and its consequences, several of which are referenced here ${ }^{4,5,6,7}$. 
The four main assumptions of the ADD model are: 1) space-time is higher dimensional, so introduce $n$ extra spacial dimensions beyond our usual three such that space-time is $3+1+n$ dimensional, 2) only allow gravity, i.e. gravitons, to propagate in all $3+1+n$ dimensions, 3 ) assume the extra dimensions are "compact", i.e. finite, so they are too small to be normally detected but large enough to impact physics, and 4) assume that Standard Model particles, e.g. quarks, gluons, photons, etc..., are confined to a $3+1$ dimensional "wall" or "brane" representing our normal world embedded in the higher dimensional space. The mechanism used in the ADD model to solve the Hierarchy Problem is to define that it doesn't exist: the reason gravity looks so weak in our $3+1$ dimensional world is that its force is diluted by existing in $3+1+n$ dimensions, so the higher dimensional "true" Planck mass, $M_{P}$, is much lower than the "apparent" Planck mass we measure in our world. As will be shown below, by adjusting the number of extra dimensions and their size, the higher dimensional Planck mass can be brought down to a level low enough, i.e. $\sim 1 \mathrm{TeV}$, to eliminate the Hierarchy Problem.

In addition to resolving the Hierarchy Problem, the ADD model leads to consequences which may be observed in nature. One of these is due to the compactification of the extra dimensions resulting in "towers" of new "particle-in-a-box" energy states called Kaluza-Klein states (named after the researchers who in the 1930's made an unsuccessful attempt to unify gravity with the electromagnetic force using extra dimensions) ${ }^{7}$. Kaluza-Klein states can be associated with a spectrum of graviton states which could influence hard scattering processes at the Large Hadron Collider (LHC) and could be Dark Matter candidates ${ }^{3}$. Another exciting consequence of the ADD model is that miniature Black Holes could exist at the greatly reduced Planck mass of around $1 \mathrm{TeV}$ and thus might be produced in $\mathrm{TeV}$ scale particle collisions, such as at the $\mathrm{LHC}^{4,5,6}$. Of course, in order for the ADD model to be a viable solution to the Hierarchy Problem, none of its consequences are allowed to conflict with existing observations. It can be shown that for $n>2$ and $M_{P} \sim 1 \mathrm{TeV}$, the ADD model does not conflict with existing astrophysical observations, cosmology, or particle accelerator experiments ${ }^{3}$ (some of these arguments will be presented later in this note). A very interesting feature of the ADD model is that it can be shown to be consistent with Type I String Theory ${ }^{1}$, which is characterized by extra dimensions, open ended strings (SM particles) stuck to a $3+1$ dimensional brane, and closed strings (gravitons) which can move freely in the extra dimensions. Because of this, detection of unique signatures predicted by the ADD model would be a strong suggestion of the validity of String Theory and thus we might be able to experimentally study String Theory effects at the $1 \mathrm{TeV}$ scale $^{8}$ !

In this spirit, the main goal of the present work is to study the possibility of detecting unique signatures of miniature Black Holes which may be created in LHC $p+p$ collisions. An estimate for $\mathrm{Pb}+\mathrm{Pb}$ collisions is also made at the end. More specifically, quantitative calculations will be carried out for mini-BH production at the LHC using a code based on the ADD model, CHARYBDIS ${ }^{9}$, and comparisons of 
charged hadron production from mini-BH decay will be made with charged hadron production from the PYTHIA QCD $\operatorname{code}^{10}$ in a particle tracking detector whose acceptance is around mid-rapidity such as in the ALICE experiment ${ }^{11}$. Since the concepts of Large Extra Dimensions and Black Holes are less familiar than other aspects of LHC physics, the interested reader can find some qualitative discussions of these concepts elsewhere ${ }^{12}$.

\section{Quantitative calculations of mini-BH production at the LHC and detection via the hadronic channel}

This section describes quantitative calculations for $\mathrm{BH}$ production and detection using a hadronic detector system with the performance and acceptance characteristics of in the LHC ALICE experiment. Unless otherwise noted, results presented refer to LHC $p+p$ collisions. Similar studies have been carried out by others for the LHC ATLAS experiment ${ }^{13,14}$. In the present study the $\mathrm{BH}$ event generator code CHARYBDIS $^{9}$ is used. CHARYBDIS represents a quantitative treatment of $\mathrm{BH}$ formation and decay for $p+p$ collisions. Three of its main features are 1) it integrates over parton distribution functions, 2) it calculates $\mathrm{BH}$ decay incorporating "grey body" factors, and 3) it is coupled to the PYTHIA QCD code ${ }^{10}$ for parton evolution and hadronization. Parton distribution functions used in this study are the same as used by Dimopoulos and Landsberg ${ }^{6}$.

Figure 1 shows the production cross section and number of BHs produced in a one-year running period at the LHC for $p+p$ at a luminosity of $10^{34} \mathrm{~cm}^{-2} \mathrm{~S}^{-1}$, and similar numbers for Run I at the Tevatron, as a function of the Planck mass from CHARYBDIS+PYTHIA. As seen, at full luminosity at the LHC for one year there are about $10^{9} \mathrm{BHs}$ produced if the Planck mass is $1 \mathrm{TeV}$, exponentially decreasing to only $1 \mathrm{BH}$ being produced in a year for $10 \mathrm{TeV}$. For the Tevatron, it is seen that only a few BHs are expected to have been produced during Run I if the Planck mass were exactly at $1 \mathrm{TeV}$, dropping to less than one $\mathrm{BH}$ for $1.1 \mathrm{TeV}$. Thus, it is not suprising that no evidence of $\mathrm{BH}$ formation has been reported so far at the Tevatron.

Figure 2 shows a comparison between transverse momentum distributions for charged hadrons at mid-rapidity at full LHC energy from CHARYBDIS+PYTHIA for two values of $M_{P}$ with that from PYTHIA alone, labeled "QCD only" (this plot is patched together from various PYTHIA runs with increasing values for the hardness of the $2 \rightarrow 2$ parton collision, each run represented by a different color). As seen for $M_{P}=1 \mathrm{TeV}$, hadrons from $\mathrm{BH}$ decays dominate over QCD processes for $p_{T}>100 \mathrm{GeV} / \mathrm{c}$, whereas for $M_{P}=5 \mathrm{TeV}$, BH decays only become important for $p_{T}>1.2 \mathrm{TeV}$. Since the ALICE experiment does not presently forsee having large-acceptance calomimetry capable of accurate particle $p_{T}$ measurements to very high values, alternative observables which are sensitive to the possibility of $M_{P}>1 \mathrm{TeV}$ are needed. Taking advantage of the large-acceptance precision tracking detectors available in ALICE, namely the combined Inner Tracking System 


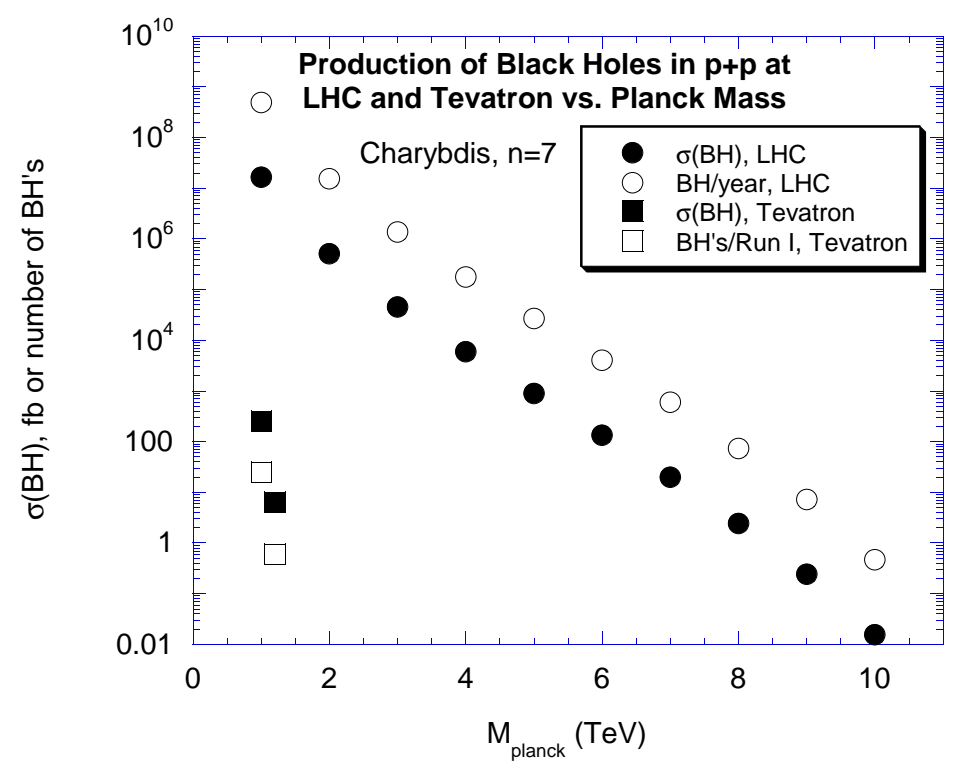

Fig. 1. Cross section and yield for mini-BH production vs. $M_{P}$ for the LHC (full luminosity and energy) and Tevatron (Run I) using CHARYBDIS.

(ITS), Time Projection Chamber (TPC) and Transition Radiation Detector (TRD) tracking, two event-by-event observables look promising for BH studies: charged multiplicity and summed $p_{T}$. The particle acceptance for charged multiplicity in rapidity and $p_{T}$ is represented by $-2<y<2$ and $p_{T}>0.1 \mathrm{GeV} / \mathrm{c}$, respectively. A reasonable acceptance which can be taken for summed $p_{T}$ per event is represented by $-0.9<y<0.9$ and $0.1<p_{T}<300 \mathrm{GeV} / \mathrm{c}$. These observables will be studied below.

The strategy of the present study will be the following: a) compare charged hadron production from BHs using CHARYBDIS+PYTHIA with QCD high$p_{T}$ processes from PYTHIA alone, b) detect these hadrons in the ALICE ITS+TPC+TRD tracking acceptance with momentum resolution effects included and c) try to find a simple triggering scheme to use for this. For all of the plots shown below for the ALICE study, the LHC will be assumed to give $\sqrt{s}=14 \mathrm{TeV}$ $p+p$ collisions at a "year-1" luminosity of $10^{31} \mathrm{~cm}^{-2} \mathrm{~s}^{-1}$. A value for the number of extra dimensions will be taken to be $n=7$. Note that for a given $M_{P}$ the results from CHARYBDIS do not depend sensitively on the exact value of $n$ used for values of this order, e.g. for $n=6$ the $\mathrm{BH}$ production cross section is within $10 \%$ of the $n=7$ case. The charged hadrons included in all plots are pions, kaons, protons, and their anti-particles. The ALICE tracking acceptance is simulated with rapidity and transverse momentum cuts, and momentum resolution effects are conservatively simulated assuming $\Delta p / p=0.16 p$, where $p$ is the particle momentum. 


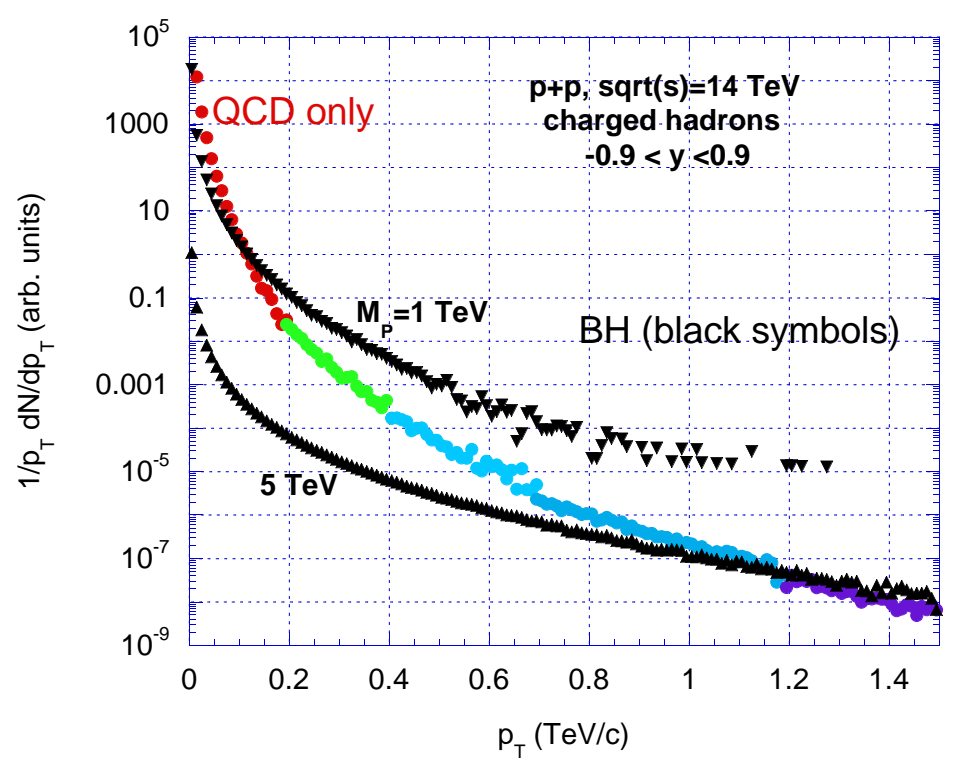

Fig. 2. Transverse momentum distributions for charged hadrons from BH decay (CHARYBDIS) compared with $2 \rightarrow 2$ hard QCD processes (PYTHIA).

Figures 3 and 4 show plots of charged hadron multiplicity per event and summed $p_{T}$ per event, respectively, for BH and QCD events in the ALICE acceptances for running a minimum-bias trigger for four months of initial LHC luminosity. The maximum data acquisition rate for $p+p$ in ALICE is taken to be $100 \mathrm{~Hz}$. For these running conditions, it is seen that only a few $\mathrm{BH}$ events will be visible above the QCD background and only for $M_{P}=1 \mathrm{TeV}$, occuring for multiplicities greater than 200 and summed $p_{T}$ greater than $0.5 \mathrm{TeV} / \mathrm{c}$.

In order to improve this situation, assume it is possible to apply a simple charged multiplicity trigger to ALICE events in the tracking acceptance $-0.9<y<0.9$ where only events with multiplicity greater than 65 are accepted. Possible detectors which could be used to implement such a trigger are the TRD and ITS, but further study of the details of this is required. Figures 5 and 6 show plots corresponding to Figures 3 and 4 above except with this multiplicity trigger. The data rate for this trigger for the LHC luminosity used is only $1 \mathrm{~Hz}$, which is $1 \%$ of the maximum data acquisition rate for ALICE $p+p$ data and which would thus have only a small impact on the overall data-taking rate for ALICE. As expected, this trigger is seen to greatly reduce the $\mathrm{QCD}$ background allowing for significant $\mathrm{BH}$ signals to be detected during this short running period. For charged multiplicity, the sensitivity to $M_{P}$ is raised to $2 \mathrm{TeV}$ and hundreds of $\mathrm{BH}$ events above background corresponding to this case are seen for multiplicities greater than 250. The situation is seen to be even better for summed $p_{T}$, where tens of thousands of $\mathrm{BH}$ events are seen above 


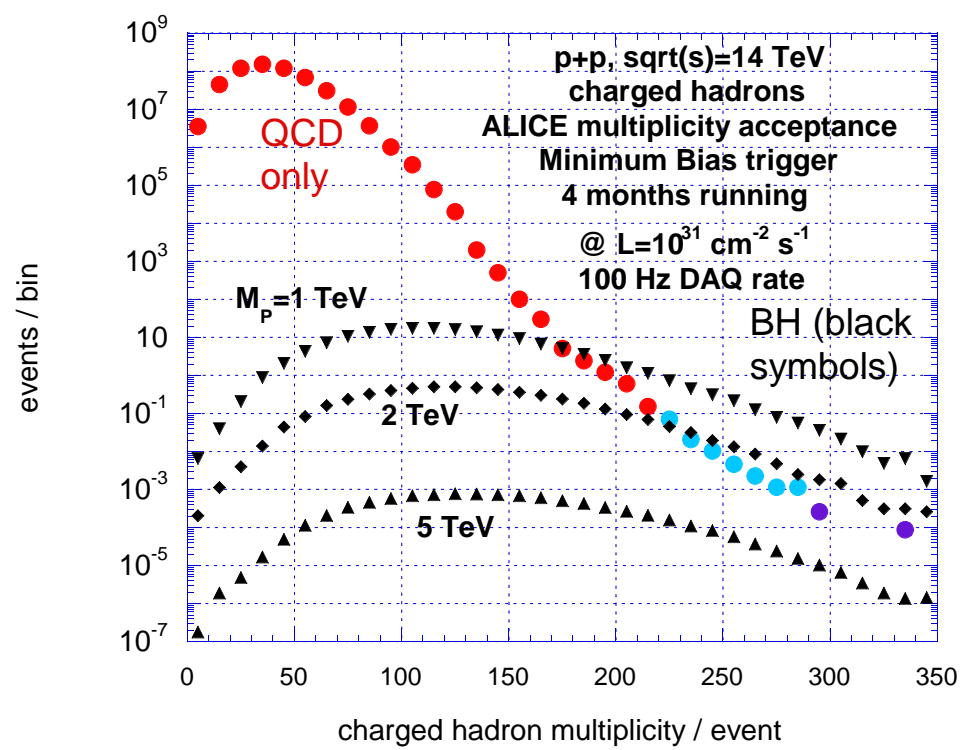

Fig. 3. Multiplicity distributions for charged hadrons for minimum bias triggering in ALICE.

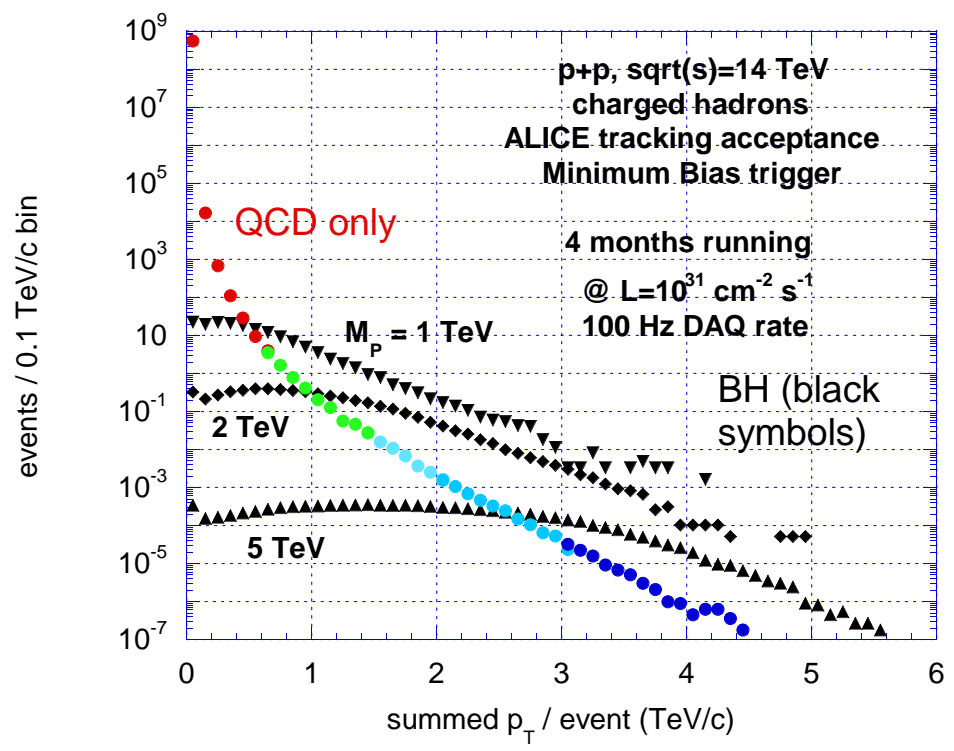

Fig. 4. Summed $p_{T}$ per event distributions for charged hadrons for minimum bias triggering in ALICE 


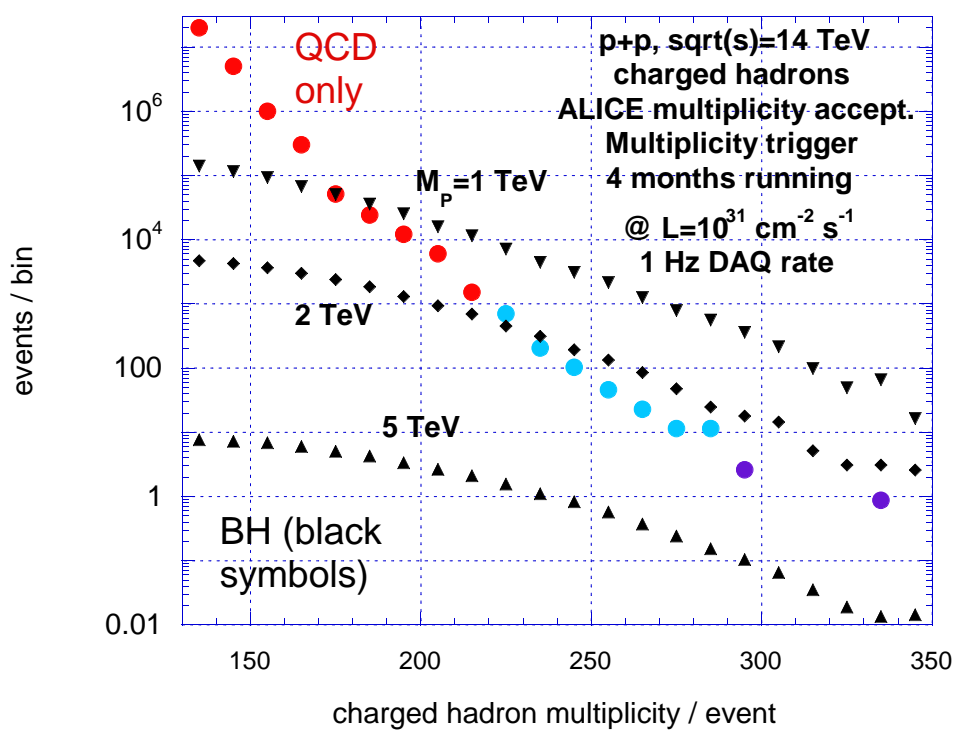

Fig. 5. Multiplicity distributions for charged hadrons using a multiplicity trigger in ALICE.

background for the $M_{P}=1 \mathrm{TeV}$ case, and tens of $\mathrm{BH}$ events may be seen above background even for the $M_{P}=5 \mathrm{TeV}$ case in this running period. The signature for $\mathrm{BH}$ creation from these simple observables is seen to be an abrupt flattening of the slope of either distribution as the transition from pure QCD to $\mathrm{BH}$ dominated charged particle production takes place. For $M_{P}<2 \mathrm{TeV}$, this flattening should be seen in both distributions in ALICE giving a redundancy to this signature. The point in multiplicity and/or summed $p_{T}$ where the flattening occurs would be related to the value of $M_{P}$ which in principle could then be determined.

It is also interesting to look at other hadronic observables which might be used as signatures for $\mathrm{BH}$ production and could be correlated with signals seen in the multiplicity and summed $p_{T}$ observables discussed above. One of these is the disappearance/suppression of high $p_{T}$ di-jets if BHs are produced ${ }^{15}$. Since BHs are thought to decay more or less isotropically by sequential Standard Model particle emissions, one would expect to observe an enhancement of mono-jets over the usual back-to-back di-jet production seen in $p+p$ collisions. One way to study this is to look at $d N / d(\Delta \phi)$ hadronic distributions, where $\Delta \phi$ is the difference in azymuthal coordinate between two hadrons in a given event. For the usual back-to-back dijet production one expects peaks in this distribution at $\Delta \phi$ of 0 and $\pi$, whereas for mono-jets one would only see a peak at 0 . Figure 7 shows a preliminary study of $d N / d(\Delta \phi)$ in the ALICE acceptance comparing BH events (CHARYBDIS) and QCD events (PYTHIA) for several different kinematical regions. A suppression of the backward peak is observed for the $\mathrm{BH}$ events and is seen to depend on the kinematical region used. 


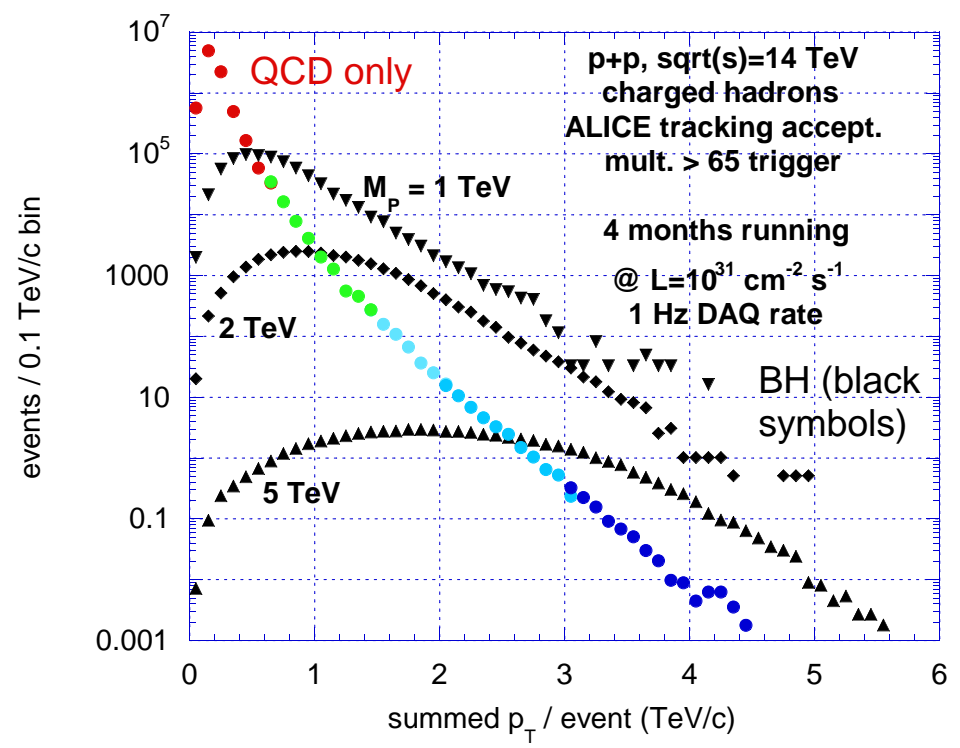

Fig. 6. Summed $p_{T}$ per event distributions for charged hadrons using a multiplicity trigger in ALICE.

Another possible signature is the direct detection of $\mathrm{BH}$ relics which are $\mathrm{BHs}$ left over at the end of the Hawking decay of mass near the higher-dimensional Planck Mass $(\sim 1 \mathrm{TeV})$ which may be stable ${ }^{16,17,18}$. A preliminary study of the ability of ALICE to detect charged $\mathrm{BH}$ relics of $2 \mathrm{TeV}$ mass is shown in Figure 8. The charged $\mathrm{BH}$ relic formation was simulated using a modified version of the CHARBYDIS code $^{19}$. Acceptance, momentum resolution and TOF resolution effects are included in this study. As seen, a $2 \mathrm{TeV}$ charged relic is easily observed in the ALICE detector.

The final topic which will be discussed is the ability to produce/detect BHs in LHC $P b+P b$ running, which will take place with lower energy, luminosity, and runtime per year compared with $p+p$. As a lower-limit estimate, assume that a $\mathrm{Pb}+\mathrm{Pb}$ collision can be represented as a superposition of free $p+p$ collisions with high $p_{T} 2 \rightarrow 2$ QCD processes. Under this assumption PYTHIA and CHARYBDIS can be used. Calculate the effective $p+p$ luminosity, $L_{e f f}$, from the expected $P b+P b$ luminosity of $L_{P b P b}=10^{27} \mathrm{~cm}^{-2} \mathrm{~s}^{-1}$, assuming $5 \%$ centrality collisions. Coincidentally, it is found that $L_{\text {eff }} \sim 10^{31} \mathrm{~cm}^{-2} \mathrm{~S}^{-1}$, the same luminosity taken in the above for ALICE $\mathrm{p}+\mathrm{p}$ running! Figure 9 shows the summed $p_{T}$ distribution for charged hadrons simulated in this way for $\mathrm{Pb}+\mathrm{Pb}$ collisions as detected in the ALICE acceptance. Only hadrons produced with $p_{T}>10 \mathrm{GeV} / \mathrm{c}$ are included in order to minimize the background from soft processes which dominate the heavyion collision. As seen, for four months of $P b+P b$ running (which represents four years of LHC running since $P b+P b$ will only be run one month per year) the case 


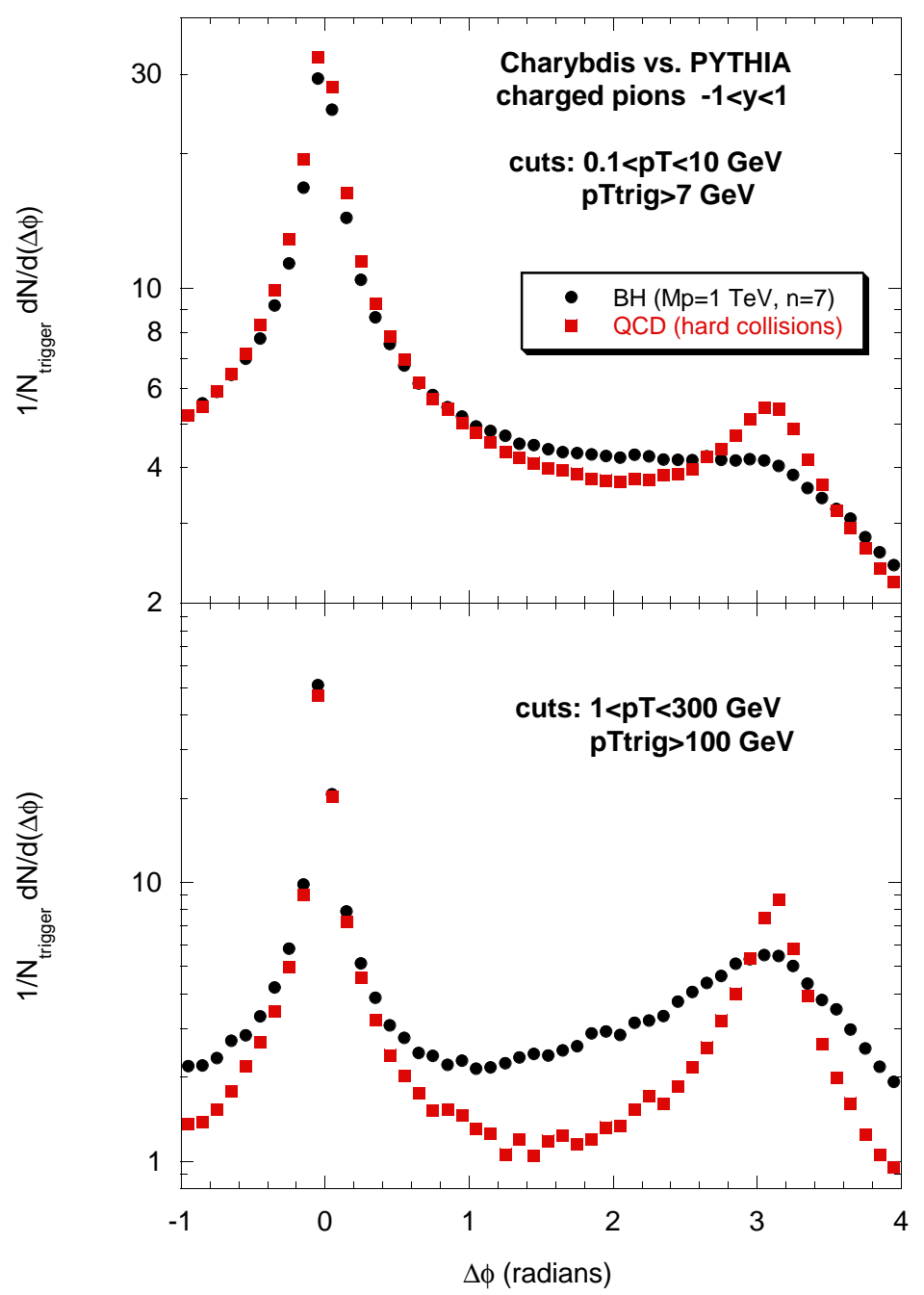

Fig. 7. $\Delta \phi$ plots for the ALICE tracking acceptance for simulated BH and QCD events for two kinematic regions.

for $M_{P}=1 \mathrm{TeV}$ can easily be detected above the QCD background, whereas for $M_{P}=2 \mathrm{TeV}$ detection is already becoming problematic.

\section{Summary}

Models with Large Extra Dimensions have exciting consequences including the possible creation of mini-BHs at the LHC. Under the proper conditions experiments such as the ALICE experiment have the capability to detect BHs from charged hadronic observables for higher-dimensional Planck masses ranging from 1 to 5 


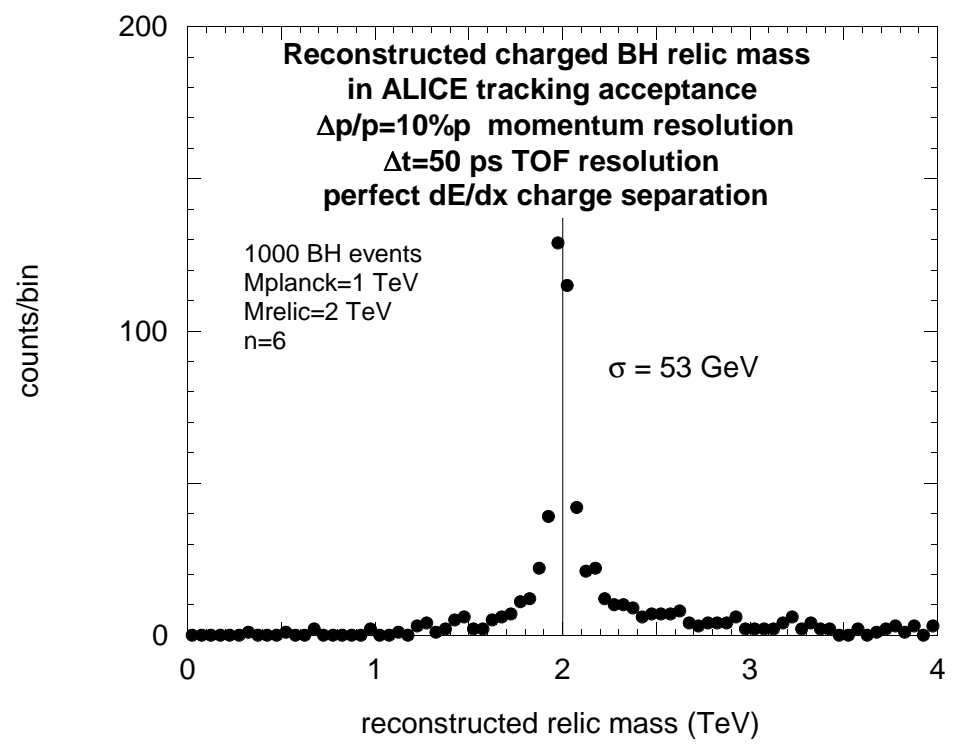

Fig. 8. Mass plot for charged relic reconstruction in ALICE. Charged BH relics with a mass of 2 $\mathrm{TeV}$ were simulated for this study.

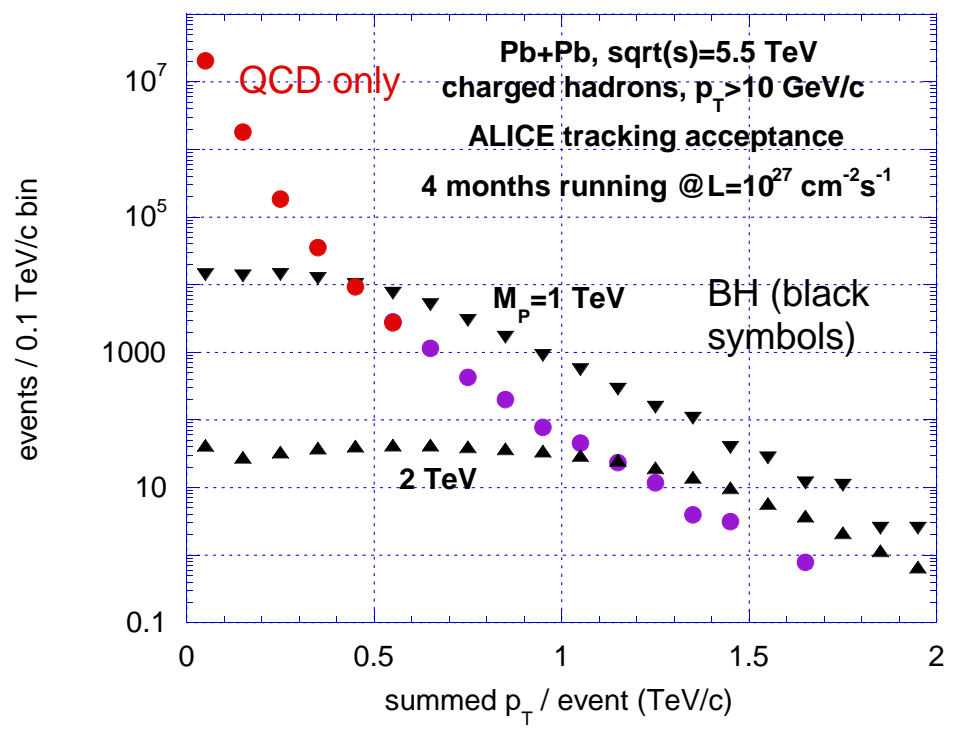

Fig. 9. Summed $p_{T}$ per event distributions for charged hadrons simulated for $P b+P b$ collisions with a central trigger in ALICE. 
$\mathrm{TeV}$ within the first four months of LHC running. A simple method for triggering on charged particle multiplicity to enhance the $\mathrm{BH}$ signal is suggested.

\section{Acknowledgements}

The authors are grateful to Chris Harris for his help in running his code CHARYBDIS. One of the authors (Humanic) would also like to acknowledge the National Science Foundation for supporting his LHC work under grant number PHY-0355007.

\section{References}

1. N. Arkani-Hamad,S. Dimopoulos,and G. Dvali, Phys.Lett. B429, 263 (1998).

2. L. Randall and R. Sundrum, Phys.Rev.Lett. 83, 3370 (1999).

3. N. Arkani-Hamad,S. Dimopoulos,and G. Dvali, Phys.Rev.D 59, 086004 (1999).

4. Steven B. Giddings and Scott Thomas, Phys.Rev.D 65, 056010 (2002).

5. T. Banks and W. Fischler, arXiv:hep-th/9906038 (1999).

6. S. Dimopoulos and G. Landsberg, Phys.Rev.Lett. 87, 161602 (2001).

7. T. G. Rizzo, arXiv:hep-ph/0409309v1 (2004).

8. E. Witten, arXiv:hep-th/0212247v1 (2002).

9. C. M. Harris, P. Richardson, and B. R. Webber, arXiv:hep-ph/0409309v1 (2004).

10. T. Sjostrand, L. Lonnblad and S Mrenna, PYTHIA 6.2 Physics and Manual, arXiv:hep-ph/0108264 (2001).

11. ALICE Collaboration, F. Carminiti et al, J. Phys.G:Nucl.Part.Phys. 30, 1517 (2004).

12. Thomas J. Humanic, ALICE internal note: ALICE-INT-2005-017.

13. J. Tanaka, T. Yamamura, S. Asai, and J. Kanzaki, ATLAS internal note: ATL-PHYS2003-037 (2003).

14. C. M. Harris, M. J. Palmer, M. A. Parker, P. Richardson, A. Sabertfakhri and B. R. Webber, arXiv:hep-ph/0411022v1 (2004).

15. H. Stöcker, arXiv:hep-ph/0605062 (2006); S. Hofmann, M. Bleicher, L. Gerland, S. Hossenfelder, S. Schwabe and H. Stöcker, arXiv:hep-ph/0111052 (2001); S. Hossenfelder, M. Bleicher and H. Stoecker, Int. J. Mod. Phys. D 13 (2004) 1453.

16. S. Hossenfelder, M. Bleicher and H. Stöcker, arXiv:hep-ph/0405031 (2004).

17. S. Hossenfelder, S. Hofmann, M. Bleicher and H. Stöcker, Phys.Rev.D66, 101502 (2002); S. Hossenfelder, S. Hofmann, M. Bleicher and H. Stöcker, Phys.Lett. B566, $233(2003)$.

18. A. Bonanno and M. Reuter, Phys.Rev.D 73, 083005 (2006).

19. B. Koch, M. Bleicher and S. Hossenfelder, JHEP 0510 (2005) 053 [arXiv:hepph/0507138]; S. Hossenfelder, B. Koch and M. Bleicher, arXiv:hep-ph/0507140. 